8. M. Gowurin, Über die Stieltjessche Integration abstrakter Funktionen, Fund. Math. vol. 27 (1936) pp. 254-268.

9. A. Grothendieck, Sur les applications linéaires faiblement compactes d'espaces de type $C(K)$, Canad. J. Math. vol. 5 (1953) pp. 129-173.

10. P. R. Halmos, Measure Theory, New York, 1950.

11. S. Kakutani, Concrete representation of abstract (M)-spaces, Ann. of Math. vol. 42 (1941) pp. 994-1024.

12. I. Singer, Les fonctionnelles linéaires sur l'espace des applications continues d'un espace de Hausdorff bicompact dans un espace de Banach (en russe), Rev. Math. Pures Appl. vol. 2 (1957) pp. 301-315.

13. - Les duals de certains espaces de Banach de champs de vecteurs, Bull. Sci. Math. vol. 82 (1958) pp. 29-40.

Universite de Bucarest, Roumanie

\title{
ON A DIVERGENT TRIGONOMETRICAL SERIES GIVEN BY STEINHAUS ${ }^{1}$
}

\section{SIOBHAN O'SHEA}

Steinhaus $[1 ; 2$, p. 283$]$ gave the series

$$
\sum_{n=2}^{\infty}(\log n)^{-1} \cos n(x-\log \log n)
$$

as an example of an everywhere-divergent trigonometric series with coefficients tending to zero. Plainly, a sine series cannot diverge everywhere, since it must converge whenever $x \equiv 0(\bmod \pi)$. There is, however, no a priori reason why a cosine series should not diverge everywhere. It is not immediately clear from Steinhaus's argument [1] whether the "cosine part" of (1), namely

$$
\sum_{n=2}^{\infty}(\log n)^{-1} \cos (n \log \log n) \cos n x
$$

has any points of convergence. Accordingly I exhibit here a class of everywhere-divergent cosine series, of which (2) is a special case.

Theorem. Suppose that $u(n) \uparrow \infty, c_{n} \downarrow 0$ as $n \rightarrow \infty$, and that there exists a sequence of positive integers $\left\{p_{n}\right\}$ such that

$$
\limsup _{n \rightarrow \infty}\left(n+p_{n}\right)\left\{u\left(n+p_{n}\right)-u(n)\right\}<\frac{1}{2},
$$

Received by the editors May 28, 1958.

1 Research sponsored by the Air Research and Development Command, United States Air Force, through its European Office, under Contract AF 61(514)-1399. 


$$
\liminf _{n \rightarrow \infty} \sum_{n+1}^{n+p_{n}} c_{r}>0
$$

Then the cosine series

$$
\sum_{n=1}^{\infty} c_{n} \cos n u(n) \cos n x
$$

diverges for all real $x$, and the sine series

$$
\sum_{n=1}^{\infty} c_{n} \sin n u(n) \sin n x
$$

diverges for all $x \neq 0(\bmod \pi)$.

The method used to prove this theorem is a refinement of that employed by Steinhaus to prove the divergence of (1). Put

$$
A_{r}(x)=\cos r u(r) \cos r x-\frac{1}{2} \cos 2 r x
$$

and suppose that

$$
u(n) \leqq x<u(n+1), \quad n+1 \leqq r \leqq n+p_{n} .
$$

Then

$$
\begin{aligned}
\left|A_{r}(x)-\frac{1}{2}\right| & =|\cos r x\{\cos r u(r)-\cos r x\}| \\
& =\left|2 \cos r x \sin \frac{1}{2} r\{x+u(r)\} \sin \frac{1}{2} r\{x-u(r)\}\right| \\
& \leqq r|x-u(r)| \\
& \leqq\left(n+p_{n}\right)\left\{u\left(n+p_{n}\right)-u(n)\right\} .
\end{aligned}
$$

By (3) there exist an integer $n_{0}$ and a number $\lambda<1 / 2$ such that this is less than $\lambda$ for all $n>n_{0}$. Thus for all $n$ and $x$ satisfying

$$
n>n_{0}, \quad u(n) \leqq x<u(n+1),
$$

we have

$$
\sum_{n+1}^{n+p_{n}} c_{r} A_{r}(x)>\left(\frac{1}{2}-\lambda\right) \sum_{n+1}^{n+p_{n}} c_{r} .
$$

Every value of $x$ satisfies (8), modulo $2 \pi$, for an infinity of values of $n$, and the left-hand side of (9) has period $2 \pi$. Thus, for every $x$, (9) is true for an infinity of $n$. Hence by (4), 


$$
\underset{n \rightarrow \infty}{\limsup } \sum_{n+1}^{n+p_{n}} c_{r} A_{r}(x)>0 .
$$

But since $c_{n} \downarrow 0$, we have for every $x$

$$
\liminf _{n \rightarrow \infty} \sum_{n+1}^{n+p_{n}} c_{r} \cos 2 r x \geqq 0
$$

and so by (7),

$$
\limsup _{n \rightarrow \infty} \sum_{n+1}^{n+p_{n}} c_{r} \cos r u(r) \cos r x>0 .
$$

By the general principle of convergence, this proves the divergence of (5).

I omit the proof of the divergence of $(6)$ for all $x \neq \equiv(\bmod \pi)$, which is similar. This proves the theorem.

To deduce from the theorem the divergence of (2) everywhere, we take

$$
u(n)=\log \log n, \quad c_{n}=(\log n)^{-1}(n \geqq 2), \quad p_{n}=\left[\frac{1}{4} \log n\right],
$$

where the square brackets denote the integer part. Then $u(n) \uparrow \infty$, $c_{n} \downarrow 0$, and the left-hand sides of (3) and (4) are both $1 / 4$. Thus (2) diverges everywhere, by the theorem. Similarly the "sine part" of (1) diverges for all $x \neq \equiv 0(\bmod \pi)$.

It is not worth while to examine in detail the order of magnitude of those sequences $\left\{c_{n}\right\}$ for which $u(n)$ and $p_{n}$ can be chosen to satisfy (3) and (4). However, it is easy to show that the choice

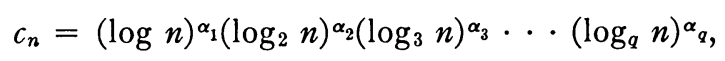

where $q$ is a fixed integer and $\log _{m}$ denotes the $m$ th iterated logarithm, is permissible if $c_{n} \downarrow 0$ and $\sum n^{-1} c_{n}=\infty$, but not otherwise.

Note added in proof, December 4,1958. If, in (5), we replace $\cos n u(n)$ by $2+\cos n u(n)$, we obtain, as may easily be seen, an everywheredivergent cosine series with non-negative coefficients tending to zero.

I wish to thank Professor P. B. Kennedy for suggesting this problem to me.

\section{REFERENCES}

1. H. Steinhaus, $A$ divergent trigonometrical series, J. London Math. Soc. vol. 4 (1929) pp. 86-88.

2. A. Zygmund, Trigonometrical series, Warsaw, 1935.

University College, Cork, Ireland 